\title{
Giant Condyloma Acuminate (Buschke-Löwenstein Tumor): A Case Report
}

\author{
Christophoros S Kosmidis, Georgios D Koimtzis*, Konstantinos Sapalidis, Efstathios Pavlidis, Stefanos \\ Atmatzidis, Vyron Alexandrou, Christina Sevva, Eleni Georgakoudi, Christina Paschalidou and Isaac I Kesisoglou
} $3^{\text {rd }}$ Surgical Department, University Hospital of Thessaloniki AHEPA, Aristotle University of Thessaloniki, Greece

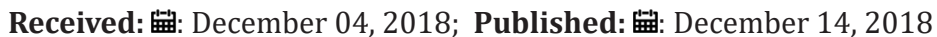

*Corresponding author: Georgios D Koimtzis, $3^{\text {rd }}$ Surgical Department, University Hospital of Thessaloniki AHEPA, Aristotle University of Thessaloniki, Thessaloniki, Greece

\begin{abstract}
Buschke-Löwenstein tumor of anorectal and perianal region is an uncommon but extremely aggressive tumor, which is often associated with human papillomavirus (HPV). Usually, it progresses slowly over the years and can be highly destructive to local tissue, while it also holds malignant potential. Herein we describe a case of a 71-year-old patient who was misdiagnosed with hemorrhoidopathy for over 20 years and was eventually treated with resection of a gigantic anorectal mass using electrocautery. In general, these tumors can reach large dimensions and possible treatment plans involve chemoradiation therapy and surgical excision. Careful follow-up of these patients is required to prevent recurrence or malignant transformation of the tumor.
\end{abstract}

Keywords: Buschke-Löwenstein Tumor; HPV Infection; Anorectal Mass

\section{Introduction}

Condyloma acuminate is a painless growth over the skin surface, which is usually, associated with human papillomavirus (HPV) types 6 and 11 [1]. Buschke-Löwenstein tumor (BLT) or giant condyloma acuminatum (GCA) is a progressively growing, cauliflower-like, locally destructive tumor that is considered to be a verrucous carcinoma, which is an intermediate state between condyloma and squamous cell carcinoma [1,2]. Patients who suffer from these tumors are susceptible to secondary infections, foul odor and fistulations. These tumors can grow rapidly and be potentially fatal to immunocompromised individuals [2, 3]. A wide, radical surgical excision of the mass is considered the primary treatment, while a close follow-up is necessary due to its frequent recurrence and malignant potential [1-3]. In this article we report the case of a 71-year-old man who was misdiagnosed for 20 years with hemorrhoidopathy and was finally treated with wide excision of a giant perianal Buschke-Löwenstein tumor.

\section{Case Report}

A 71-year-old male patient presented in our outpatient clinic complaining about a perianal mass accompanied by scarce episodes of rectal bleeding. His symptoms initially began 20 years ago but at the time he was misdiagnosed with hemorrhoidopathy and was treated accordingly. Twelve years ago he suffered from mycordial infraction and was treated with a double percoutaneous transluminal coronary angioplasty. In the same year he was diagnosed with abdominal aortic aneurysm and was treated with endovascular repair. Four year ago he was diagnosed with b-cell lymphoma and is under medical treatment until now. Clinical examination of the anorectal region revealed a gigantic tumor, which was a case of GCA of Buschke-Löwenstein (Figure 1). Under general endotracheal anesthesia and in lithotomy position the patient was subjected to wide, radical excision of the mass using monopolar diathermy and an electrothermal bipolar vessel sealing device (LigaSureTM) (Figure 2). The specimen (Figure 3) was sent for histopathological examination. The patient had an uneventful post-operative course and was discharged on the first postoperative day.

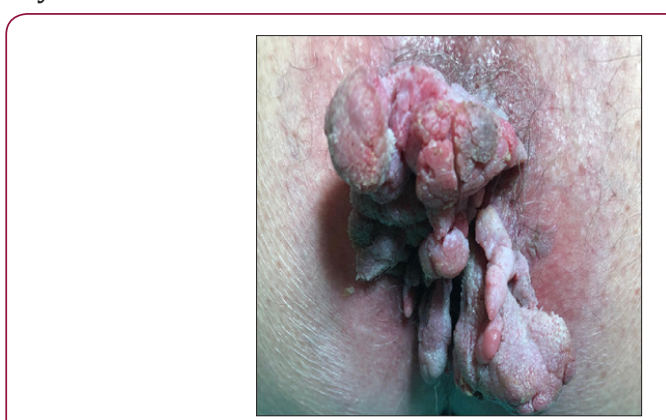

Figure 1: Anorectal Buschke-Löwenstein Tumor. 


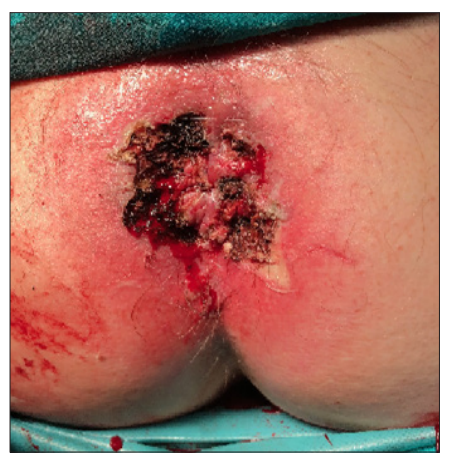

Figure 2: Wide excision of the tumor.

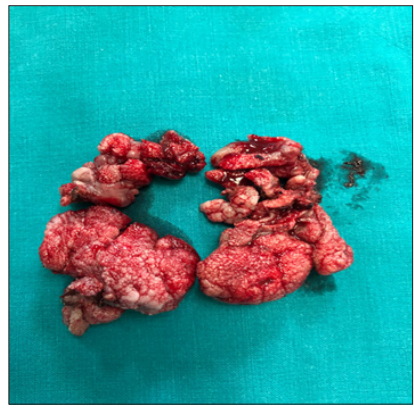

Figure 3: The specimen after being excised.

\section{Discussion}

BLT is a sexually transmitted disease triggered by HPV, more frequently subtypes 6 and $11[2,4]$. Its incidence is $0.1 \%$ in the general population with a $2.3: 1$ male to female ratio [2,5]. It usually manifests clinically as a palpable mass that can be accompanied by pain, fistulas, bleeding and pruritus [4]. In the majority of the cases it starts from long-standing condylomata that can reach up to $10-15 \mathrm{~cm}$. The evolution period usually ranges from 2.8 to 9.6 years [1]. The most important risk factors include anal receptive sex, immunosuppression, HIV infection, chronic irritation and poor personal hygiene [1]. BLT is sometimes associated with severe morbidity due to its invasive growth which can result into local tissue destruction and occlusion of the rectum, especially in cases of malignant transformation into squamous cell carcinoma [6,7]. Histologically BLT has benign appearance with negligible cellular atypia, papillomatosis, koilocytosis and epithelial hyperplasia [1,2]
However, these histological features are very similar to those of verrucous carcinoma and some authors do not differentiate between these diseases [4]. Nonetheless, BLT has low metastatic potential [4]. Because of its scarceness and lack of controlled studies, there are no gold standards in managing BLT [2,7]. Therapeutic options include topical agents (podophillin, bleomycin, interferon and 5-fluorouracil), systemic therapy with chemoradiation, cryotherapy with liquid nitrogen, use of carbon laser and surgical management [2,7]. Surgical resection with wide tumor-free surgical margins seems to be the treatment of choice due to BLT's high rate of recurrence (66\%) and malignant transformation (56\%) [1]. The effectiveness of adjuvant therapy remains under study [7].

\section{Conclusion}

In this article we present a case of a patient who presented with a large anorectal mass that was proven to be a BLT. He was initially misdiagnosed with hemorrhoidopathy twenty years ago. This delay in the correct diagnosis might affect the patient's life quality as it increases the risk of recurrence and malignant transformation.

\section{References}

1. Bastola S, Halalau A, Kc O, Adhikari AA (2018) Gigantic Anal Mass: Buschke-Löwenstein Tumor in a Patient with Controlled HIV Infection with Fatal Outcome. Case Rep Infect Dis 2018: 7267213.

2. Kim HG, Kesey JE, Griswold JA (2018) Giant anorectal condyloma acuminatum of Buschke-Löwenstein presents difficult management decisions. J Surg Case Rep 2018(4): rjy058.

3. Venter F, Heidari A, Viehweg M, Rivera M, Natarajan P, et al. (2018) Giant Condylomata Acuminata of Buschke-Lowenstein Associated with Paraneoplastic Hypercalcemia. J Investig Med High Impact Case Rep 6: 2324709618758348.

4. Araújo Paulo Sérgio Ramos de, Padilha Carlos Eduardo Guimarães, Soares Melina Ferraz (2017) Buschke-Lowenstein tumor in a woman living with HIV/AIDS. Rev Soc Bras Med Trop 50(4): 577-577.

5. Patel R, Kaloucava S (2017) A case of penile Buschke-Lowenstein tumor in a developing country. Clin Case Rep 5(3): 257-259.

6. Grodner C, Henn A, Lelièvre JD, Gallien S (2016) Successful improvement of Buschke-Löwenstein tumour in an HIV-infected patient with antiretroviral therapy alone. BMJ Case Rep 2016: bcr2016217753.

7. Badiu DC, Manea CA, Mandu M, Chiperi V, Marin IE, et al. (2016) Giant Perineal Condyloma Acuminatum (Buschke-Lowenstein Tumour): A Case Report. Chirurgia (Bucur) 111(5): 435-438.
ISSN: 2574-1241

DOI: $10.26717 / B J S T R .2018 .12 .002216$

Georgios D Koimtzis. Biomed J Sci \& Tech Res

This work is licensed under Creative Commons Attribution 4.0 License

Submission Link: https://biomedres.us/submit-manuscript.php

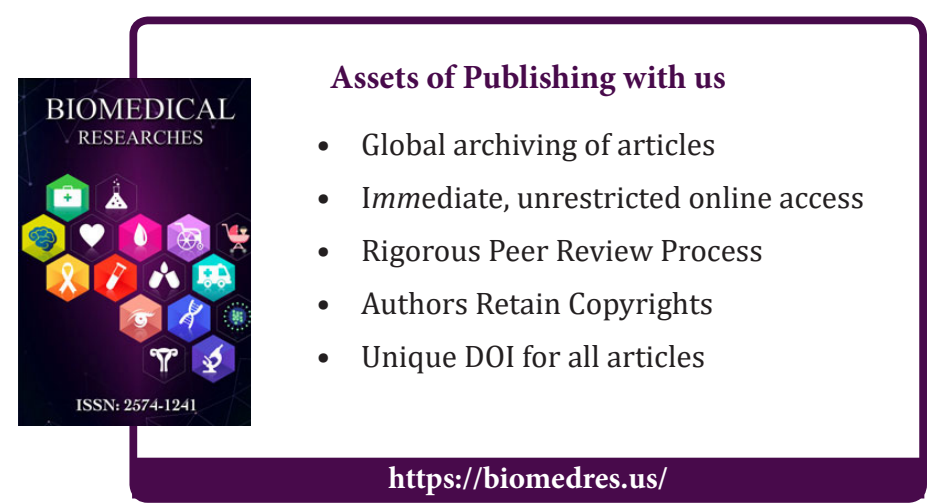

Cite this article: Kosmidis C K, Koimtzis G D, Sapalidis K, Pavlidis E, Atmatzidis S et al. Giant Condyloma Acuminate (Buschke-Löwenstein Tu- 